\title{
Endoscopy waiting times and impact of the two week wait scheme on diagnosis and outcome of upper gastrointestinal cancer
}

\author{
T Spahos, A Hindmarsh, E Cameron, M R Tighe, L Igali, D Pearson, M Rhodes, M P N Lewis
}

Postgrad Med J 2005;81:728-730. doi: 10.1136/pgmj.2004.031104

The NHS has introduced the two week wait scheme to detect upper gastrointestinal cancers at an early stage and improve survival rates The aim of this study was to assess the impact of this scheme and changes in endoscopy waiting times on tumour stage and resection rates over a four year period. Data were analysed prospectively for all patients diagnosed with oesophagogastric cancer between September 1998 and September 2002 and from those referred under the two week wait scheme since its introduction in 2000 . Of those tumours diagnosed by this scheme (15\%) only $5 \%$ were early disease (stage 1 or 2). Patients with early cancer, mainly diagnosed by routine gastroscopy, do not present with symptoms meeting the two week wait criteria. An increase in the resection rates for early disease will most probably be seen with a reduction in routine endoscopy waiting times.

$\mathrm{E}$ arly diagnosis and treatment of cancer is widely accepted to improve prognosis in oesophagogastric cancer. The past few years have seen increased political and public pressure to improve the service for cancer patients in the UK. This has followed concerns that theUK lags behind Western Europe in terms of cancer survival. This is particularly pronounced in gastric cancer. ${ }^{1}$ One possible reason for poor cancer survival in the UK is the delayed access to specialist clinical and diagnostic services within the NHS for patients with suspected cancer resulting in delayed treatment (the average time to see a consultant has been seven weeks, with three quarters of people getting an outpatient appointment within 13 weeks of the GP requesting one). ${ }^{2}$

The current UK government plan to tackle this issue has led to the introduction of the two week wait scheme. This measure was included in the government white paper The new NHS—Modern, Dependable published in December 1997. This proposes that patients with suspected cancer should be able to see a specialist within two weeks of their GP referring. These arrangements were implemented nationally for upper gastrointestinal cancers in July 2000.

There are about 17000 new cases of oesophageal and gastric cancer each year in the UK. These cancers have a particularly poor survival rate in part because of late stage at diagnosis. To assist appropriate referrals by GPs a list of guidelines and criteria for urgent referral (box 1) has been developed by the Department of Health (DoH), based on the published literature and the unpublished audits of symptoms in patients presenting with upper gastrointestinal (GI) cancer.

The two week wait scheme for oesophagogastric cancers was introduced to our institution in September 2000. Significant service improvements at the same time permitted the introduction of two full time nurse endoscopists. Our aim was to assess the validity of the referral guidelines in terms of new cancer diagnosis within the patient population referred under the scheme. We have also looked at the impact of the two week wait initiative and the changes in endoscopy times on tumour stage at resection and cancer resection rates over a four year period.

\section{METHODS}

We analysed prospectively collected data on all oesophagogastric cancer patients presenting to the Norfolk and Norwich University Hospital upper gastrointestinal surgical department for two years before and after the introduction of the two week wait scheme. Specifically we looked at resection rate, tumour stage at resection, presenting symptoms, method of referral, and endoscopy waiting times.

To assess the number of new gastric and oesophageal cancers detected through the two week initiative as a proportion of all new cancers detected during the study period the details of all other patients with a new diagnosis of oesophageal or gastric cancer were obtained from the cancer audit database. These patient details were matched by hospital number with the theatre information database (ORSOS) to determine the resection rate among this group of patients and also permit comparison with the two week wait initiative group. The clinical notes were obtained for all patients undergoing resection.

Standard practice after referral, either on or off two week wait scheme was to arrange for an initial gastroscopy for diagnosis. After diagnosis all patients were staged by multislice computed tomography, endoscopic ultrasound

\section{Box 1 Urgent referral guidelines}

- Dysphagia (any age)

- Jaundice

- Upper abdominal mass

- Dyspepsia plus one or more of the following: significant family history; pernicious anaemia; peptic ulcer surgery over 20 years ago; known dysplasia, atrophic gastritis, intestinal metaplasia

- Dyspepsia in a patient aged 55 years or more with onset of less than one year or continuous symptoms since onset

- Dyspepsia combined with one or more of the following "alarm" symptoms:

- weight loss

- proven anaemia

- vomiting 


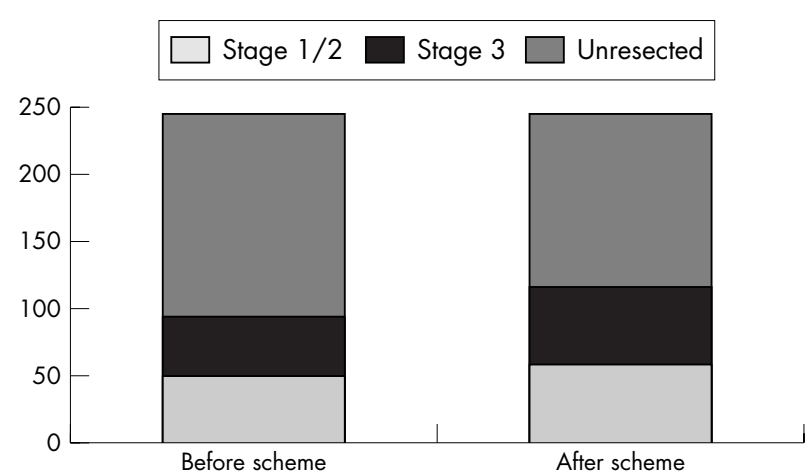

Figure 1 Changes in resection rates by histopathological stage in the two years before the introduction of the two week scheme and two years after.

(oesophageal and cardia tumours only), and laparoscopy (potentially resectable gastric tumours only). Stage 2 and 3 oesophageal and cardia tumours were offered neoadjuvant chemotherapy. Surgery was offered to patients staged 1-3 providing adequate fitness. Cancer stage was confirmed from histological reports after resection and also using both the histology and radiology (ISIS) database. Results were divided into two years before and two years after introduction of the two week wait scheme and analysed statistically where appropriate using $\chi^{2}$ test.

\section{RESULTS}

During the four year period a total of 494 patients were diagnosed with oesophagogastric cancer; 247 before the introduction in the two years and exactly 247 after.

Before the introduction of the scheme the curative resection rate was 38\% (94 of 247). After scheme introduction this increased to $48 \%$ ( 118 of 247 ) (odds ratio $1.48,95 \%$ CI 1.03 to 2.11) (fig 1). The number of curative resections for early stage disease (stage 1 and 2) rose correspondingly from 47 to 58 .

In the two years after the scheme introduction there were 623 referrals under the two week wait scheme for oesophagogastric cancer of which 38 were found to have cancer $(6 \%$ of two week wait referrals, $15 \%$ of cancers). Of the 38 patients diagnosed with oesophagogastric cancer under the two week wait scheme only two $(5 \%)$ had early stage disease compared with 56 (27\%) diagnosed outside scheme.

Examining presenting symptoms in 55 patients with early disease showed that 19 patients (35\%) had symptoms that fulfilled the criteria of the two week wait scheme. However, only two of these patients were referred via this route. Box 2 shows the presenting symptoms in patients with early disease.

Box 2 Presenting symptoms in patients with early disease

- 19 patients with urgent referral criteria

- 6 urgent admissions with frank bleeding

- 13 with anaemia only

- 10 with dyspepsia only

- 1 with vomiting only

- 3 with combinations (weight loss, anaemia, vomiting)

- 1 from screening

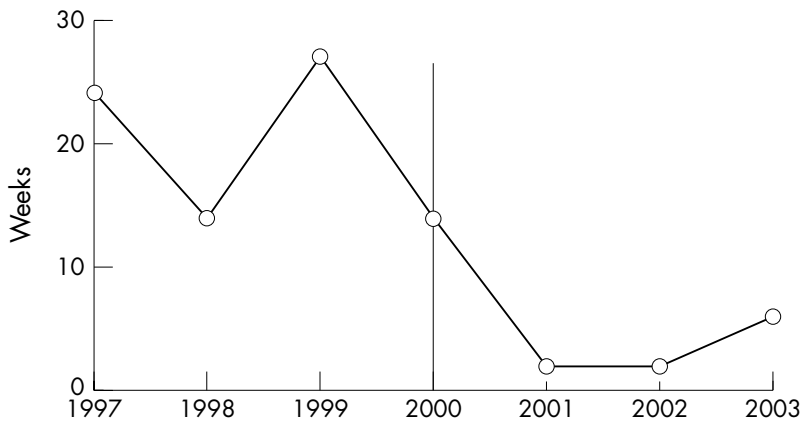

Figure 2 Changes in routine outpatient gastroscopy waiting time 1997-2003. Nurse endoscopists were introduced in 2000.

There was a steady decline in routine endoscopy waiting times during the same period. This corresponded with a rise in the number of endoscopies performed (fig 2) after the introduction of nurse endoscopists.

\section{DISCUSSION}

We have seen a substantial increase in the curative resection rate for oesophagogastric tumours in our unit since the introduction of the government's two week wait scheme. This has corresponded with a rise in the early stage cancers over the same period. However, it would seem that this link is only circumstantial. From the large number of patients referred under the scheme disappointingly only two had early stage disease. This suggests that the two week wait scheme is probably not the reason for the observed improved resection rate.

Early diagnosis and subsequently definitive treatment were facilitated by early referral by GPs and a multidisciplinary team approach, but primarily by the introduction of nurse endoscopists, which coincided with introduction of the two week wait scheme. This led to a considerable reduction in routine endoscopy waiting times and consequently to an increased resection rate.

The signs and symptoms listed on the two week wait scheme for upper GI cancers are those commonly seen with late stage disease rather than early. This would explain why most cancers diagnosed in our unit through the scheme were late stage. In contrast, although alarm symptoms consistently predict serious upper GI abnormality, a significant proportion of patients with upper GI cancer will have no alarm symptoms. ${ }^{3}$

Our findings support those of previously published audits at other units. ${ }^{4-6}$ The poor cancer pick up rate $(4 \%-15 \%)$ might reflect poor specificity of the referral criteria or a high degree of inappropriate referrals. ${ }^{7}$ These few cases of cancer detected under the scheme have advanced disease with most of the new cancers diagnosed outside the scheme. Furthermore, the "two week rule" has led in many cases to a significant increase in referrals for endoscopy with a potential adverse effect on the routine non-urgent waiting times. ${ }^{8}$

Improving outcomes in patients with oesophagogastric cancer in the UK may be achieved by earlier diagnosis; patients with stage 1 or 2 disease are most likely to benefit from surgery. The two week wait scheme would only have identified $35 \%$ of our patients with early stage disease even if scheme utilisation was $100 \%$.

This work suggests that the two week wait scheme is unlikely to improve survival figures for oesophagogastric cancers in the UK. It may well be that improving resources for routine endoscopy will be of more benefit. 


\section{Authors' affiliations}

T Spahos, A Hindmarsh, D Pearson, M Rhodes, M P N Lewis, Division of Upper Gastrointestinal Surgery, Department of General Surgery, Norfolk and Norwich University Hospital NHS Trust, UK

E Cameron, M R Tighe, Division of Gastroenterology, Department of General Medicine, Norfolk and Norwich University Hospital NHS Trust L Igali, Department of Pathology, Norfolk and Norwich University Hospital NHS Trust

Funding: none.

Conflicts of interest: none.

Correspondence to: MrT Spahos, Division of Upper Gastrointestinal Surgery, Department of General Surgery, Norfolk and Norwich University Hospital, Colney Lane, Norwich NR4 7UY, UK; theospahos@ hotmail.com

Submitted 30 November 2004

Accepted 13 January 2005

\section{REFERENCES}

1 EUROCARE-3. EUROCARE-3 summary: cancer survival in Europe at the end of the 20th century. Ann Oncol 2003; 14(suppl 5):v128-49.

2 Department of Health. Tough new monitoring regime for out-patients unveiled as new weapon in the war on NHS waiting. Press release, 6 Sep, 1999

3 Chaudhary R, Parham D, Catnach S, et al. How does the receiving clinician's assessment of urgency of endoscopy affect waiting times and endoscopic findings? Gut 2002;50:a96-107.

4 Melleney E M-A, Willoughby CP. Audit of a nurse endoscopist based one stop dyspepsia clinic. Postgrad Med J 2002:78:161-4.

5 Loehry JK, Smith TR, Vyas SK. Achieving the 'two week standard' for suspected upper $\mathrm{Gl}$ cancers: continuing pain with minimal gain: a retrospective audit. Gut 2002;50:a96-107.

6 Lassman DJ, Elliott J, Taylor A, et al. Service implications and success of the implementation of the two-week referral criteria for upper $\mathrm{Gl}$ cancers in a district general hospital. Gut 2002;50:a63-7.

7 Bassi A, Sturgess R, Bodger K. Rapid access upper Gl cancer service versus open access endoscopy: impact of the 'two week rule'. Gut 2002:50:a96-107.

8 Boulton-Jones JR, Gamble S, Goddard WP, et al. The impact and clinical appropriateness of the two week wait scheme for suspected cancer. Gut 2002;50:a107-11.

\section{bmjupdates+}

bmiupdates+ is a unique and free alerting service, designed to keep you up to date with the medical literature that is truly important to your practice.

bmjupdates+ will alert you to important new research and will provide you with the best new evidence concerning important advances in health care, tailored to your medical interests and time demands.

Where does the information come from?

bmjupdates+ applies an expert critical appraisal filter to over 100 top medical journals A panel of over 2000 physicians find the few 'must read' studies for each area of clinical interest

Sign up to receive your tailored email alerts, searching access and more...

www.bmjupdates.com 